\title{
KÄHLER METRICS ON FIBERED MANIFOLDS ${ }^{1}$
}

\author{
PAUL C. YANG
}

\begin{abstract}
A Bochner type integral identity is used to derive a nonfibration theorem for complex manifolds with negative holomorphic bisectional curvature. As an application, a classification of fibered compact Kähler surfaces with nonpositive bisectional curvature is given.
\end{abstract}

0. Introduction. In [1], Goldberg and Kobayashi introduced the notion of holomorphic bisectional curvature in order to study compact Kähler manifolds with positive tangent bundle. In this paper we derive a Bochner-type formula for the variation of volume of a factor in a product manifold involving an integral of this curvature function. As a consequence, the following theorem is derived in $\S 1$.

THEOREM. If $F \rightarrow M \rightarrow N$ is a holomorphic fiber bundle, where $F$ is compact, then $M$ does not admit a Kähler metric of negative holomorphic bisectional curvature. In fact, a Kähler metric on $M$ with nonpositive holomorphic bisectional curvature induces a Kähler metric on $N$ making $M \rightarrow N$ a Riemannian submersion, and the metric on $M$ is, over each trivialization, a product metric.

In $\$ 2$ we apply this observation to compact Kähler surfaces to obtain

COROLlary 1. If $M$ is a compact Kähler surface with nonpositive bisectional curvature $K$, and such that $K\left(\sigma, \sigma^{\prime}\right)<0$ for all holomorphic planes $\sigma, \sigma^{\prime}$ at some $p \in M$, then $M$ is an algebraic surface of general type.

Next we study the fibered Kähler surfaces with nonpositive bisectional curvature. The result is the following classification.

COROLlary 2. If $M$ is a compact fibered Kähler surface with nonpositive holomorphic bisectional curvature, then $M$ is one of the following:

(1) $M$ is flat, i.e., $M=E \times E^{\prime}$, the metric product of two elliptic curves with flat metric.

(2) $M$ is a torus bundle over a curve $C$ of genus greater than one. In this case, the universal cover of $M$ is $\mathbf{C} \times D$ endowed with the product of the flat metric on $\mathbf{C}$ and a complete metric of nonpositive curvature on D.

(3) $M$ is fibered over a torus with fiber a curve of genus greater than one. In this case the universal cover is $D \times \mathbf{C}$ endowed with the product of the flat

Received by the editors July 16, 1976.

AMS (MOS) subject classifications (1970). Primary 53C55; Secondary 32K05.

${ }^{1}$ Research supported by NSF Grant MPS 75-05270. 
metric on $\mathbf{C}$ with a complete metric of nonpositive curvature on $D$.

(4) $M$ is a quotient of $C \times D$ where $C$ is a curve of genus greater than one. In this case, $C$ is isometric to the fiber in $M$, and $C \times D$ is endowed with a product of a metric of nonpositive curvature on $C$ with a complete metric of nonpositive curvature on $D$.

1. Preliminary and proof of theorem. We follow the notations of Kobayashi and Nomizu [2]. Let $z^{1}, \ldots, z^{n}, z^{n+1}(=w)$ be local coordinates in a neighborhood $U \times D, U$ a domain in $\mathbf{C}^{n}, D=$ unit disc in $\mathbf{C}$. Let

$$
\Phi=(-2 i) \sum_{1<A, B<n+1} g_{A \bar{B}} d z^{A} \wedge d \bar{z}^{B}
$$

be the Kähler form of a Kähler metric on $U \times D$. The volume form of a level hypersurface $M_{c}=\{(z, w) \in U \times D \mid w=c\}$ is given by $\Phi_{c}^{n}=\Phi_{c} \wedge \cdots \wedge$ $\Phi_{c}$, where

$$
\Phi_{c}=(-2 i) \sum_{1<i, j<n} g_{i j}(z, c) d z^{i} \wedge d \bar{z}^{j}
$$

We compute the derivative of $\Phi_{w}^{n}$ with respect to $w$ :

$$
\begin{aligned}
\frac{\partial^{2}}{\partial w \partial \bar{w}} \Phi_{w}^{n}= & n \Phi_{w}^{n-1} \wedge(-2 i)\left(\sum_{1<i, j<n} \frac{\partial^{2} g_{i j}}{\partial w \partial \bar{w}} d z^{i} \wedge d \bar{z}^{j}\right) \\
& +n(n-1) \Phi_{w}^{n-2} \wedge \frac{\partial \Phi_{w}}{\partial w} \wedge \frac{\partial \Phi_{w}}{\partial \bar{w}} .
\end{aligned}
$$

The components of the curvature tensor being given by

$$
K_{i j k \bar{l}}=\frac{\partial^{2} g_{i j}}{\partial z^{k} \partial \bar{z}^{l}}-\sum g^{\bar{\alpha} \beta} \frac{\partial g_{i \bar{\alpha}}}{\partial z^{k}} \frac{\partial g_{\bar{j} \beta}}{\partial \bar{z}^{l}},
$$

we may write

$$
\begin{aligned}
\frac{\partial^{2} \Phi_{w}}{\partial w \partial \bar{w}}= & n \Phi_{w}^{n-1} \wedge(-2 i)\left(\sum_{1<i, j<n} K_{i j n+1} \overline{n+1} d z^{i} \wedge d \bar{z}^{j}\right) \\
& +n \Phi_{w}^{n-1} \wedge(-2 i)\left(\sum_{\substack{1<i, j<n \\
1<A, B<n+1}} g^{\bar{A} B} \frac{\partial g_{i \bar{A}}}{\partial w} \frac{\partial g_{j B}}{\partial \bar{w}} d z^{i} \wedge d \bar{z}^{j}\right) \\
& +n(n-1) \Phi_{w}^{n-2} \wedge \frac{\partial \Phi_{w}}{\partial w} \wedge \frac{\partial \Phi_{w}}{\partial \bar{w}}
\end{aligned}
$$

Proof of Theorem. Let $F \rightarrow M \rightarrow N$ be as above, $F \times D \subset M$ be a local trivialization of $M$ over a disc in $N$. Let $U$ be a coordinate neighborhood of $F$; then we are in the setup discussed in the preliminary. The function $v$ : $D \rightarrow \mathbf{R}^{+}$given by $v(w)=$ volume of fiber over $w$ is constant (since Kähler submanifold minimizes volume in its homology class); hence $\left(\partial^{2} v / \partial w \partial \widehat{w}\right)(w)$ $=0$ for all points in $D$; thus if $M$ has nonpositive holomorphic bisectional curvature $\left(K_{\alpha \bar{\alpha} \beta \bar{\beta}} \geqslant 0\right)$, all three integrands appearing in $\partial^{2} v(w) / \partial w \partial \bar{w}=$ 
$\int_{M_{w}} \partial^{2} \Phi_{w}^{n} / \partial w \partial \bar{w}$ are nonnegative. Hence, each must vanish identically. As a consequence we have

$$
\partial g_{i \bar{A}} / \partial w=\partial g_{w \bar{A}} / \partial z^{i}=\partial g_{\bar{j} B} \partial \bar{w}=\partial g_{\bar{w} B} / \partial \bar{z}^{j}=0,
$$

which are precisely the desired conclusions.

2.

Proof of Corollary 1. We examine the Kodaira classification of surfaces [3]. Recalling the fact that holomorphic sectional curvature decreases on a complex submanifold of a Kähler manifold, we may deduce from the GaussBonnet Theorem that $M$ does not contain $P^{1}$. In the Kodaira list of surfaces, with the exception of the algebraic surfaces of general type, all surfaces either contain a $P^{1}$ or have vanishing first Chern class or are elliptic bundles which are ruled out by our Theorem.

Proof of Corollary 2. Suppose $M \rightarrow C$ is a Kähler surface fibered over a curve $C$, with nonpositive bisectional curvature, then the Theorem implies that the fibers are totally geodesic submanifolds of $M$, hence, in particular, the curvature $K$ of the fiber in the induced metric agrees with the ambient holomorphic sectional curvature; we consider two cases. First, $K \equiv 0$ in one fiber (hence $\equiv 0$ for all fibers since the fibers are isometric). This implies $\underset{\sim}{F}$ is an elliptic curve. Consider the universal cover $\pi: \tilde{C} \rightarrow C$; then $\pi^{*} M \rightarrow \tilde{C}$ is again a Kähler surface fibered over $\tilde{C}$ with nonnegative holomorphic bisectional curvature, hence the induced metric is a product metric. In the case $\tilde{C}=\mathbf{C}$ we can conclude by the Gauss-Bonnet Theorem that the metric on $\mathbf{C}$ must be the usual flat metric, and this is statement (1). If $\tilde{C}=D$ we have (2).

Second, $K \neq 0$, hence $K<0$ somewhere, therefore the fiber $F$ is a curve of genus greater than one. Suppose $\tilde{C}=\mathbf{C}$ then again $C$ is an elliptic curve with flat metric. Let $\pi: \mathbf{C} \rightarrow C$ be the covering map; then our Theorem applied to $\pi^{*} M$, the induced fiber bundle over $\mathbf{C}$, is a Riemannian product since $\mathbf{C}$ is simply connected. This is the case covered by statement (3).

The remaining possibility is $\tilde{C}=D$; hence we have statement (4).

\section{REFERENCES}

1. S. I. Goldberg and S. Kobayashi, Holomorphic bisectional curvature, J. Differential Geometry 1 (1967), 225-233. MR 37 \#3485.

2. S. Kobayashi and K. Nomizu, Foundations of differential geometry, Vol. II, Interscience, New York, 1969. MR 38 \#6501.

3. K. Kodaira, On compact analytic surfaces, Analytic Functions, Princeton Univ. Press, Princeton, N. J., 1960, pp. 121-135. MR 25 \#3939.

Department of Mathematics, Rice University, Houston, Texas 77001

Curent address: School of Mathematics, The Institute for Advanced Study, Princeton, New Jersey 08540 